\title{
Nachrichten der Gesellschaft für Kinder- und Jugendrheumatologie
}

\section{Ausblick auf ein neues Jahr}

Ein neues Jahr mit neuen Herausforderungen liegt vor uns, der Veranstaltungskalender ist bereits gut gefüllt ( $\triangleright$ Tab. 1). Viele bewährte Veranstaltungsformate stehen auf der Agenda, angefangen vom GarmischPartenkirchener Symposium für Kinderund Jugendrheumatologie im Januar bis zum Hamburger Sklerodermie Symposium am Ende des Jahres. Hermann Girschick ist diesjähriger Tagungspräsident der Jahresta- gung der DGJK im September in Berlin. Aus diesem Anlass findet die 30. Jahrestagung der GKJR, deren Tagungspräsident Tilmann Kallinich ist, in diesem Jahr gemeinsam mit der DGKJ statt. Zu den inhaltlichen Schwerpunkten Bewegung, Entzündungsvorgänge/Inflammation, genetische Diagnostik und darauf basierende Therapien und digitaler Wandel in der Medizin werden interessante Workshops und Symposien angebo- ten. Bereits im April lädt Martin Kirschstein als Tagungspräsident der 69. Jahrestagung der Norddeutschen Gesellschaft für Kinderund Jugendmedizin nach Celle ein.

Auch in diesem Jahr werden wieder zahlreiche lokale Veranstaltungen, die noch nicht alle im Veranstaltungskalender aufgeführt sind, das Fort- und Weiterbildungsangebot bereichern. Darüber hinaus wird ein 3. Stra-

- Tab. 1 Veranstaltungskalender 2020.

\begin{tabular}{|c|c|c|c|}
\hline Veranstaltungstitel & Datum & Ort & Veranstalter \\
\hline $\begin{array}{l}\text { 45. Garmisch-Partenkirchener Symposium für Kinder- und } \\
\text { Jugendrheumatologie }\end{array}$ & 17./18.01.2020 & $\begin{array}{l}\text { Garmisch- } \\
\text { Partenkirchen }\end{array}$ & $\begin{array}{l}\text { Deutsches Zentrum für Kinder- und Jugend- } \\
\text { rheumatologie }\end{array}$ \\
\hline $\begin{array}{l}\text { Workshop Ped-MUSTM: Gelenk-Ultraschall in der Kinder- } \\
\text { rheumatologie }\end{array}$ & 24./25.01.2020 & Sendenhorst & St. Josef-Stift \\
\hline $\begin{array}{l}\text { Symposium Süddeutscher Kinderrheumazentren: Biosimilare im } \\
\text { Fokus der pädiatrischen Rheumatologie }\end{array}$ & 19.02.2020 & Stuttgart & $\begin{array}{l}\text { Zentrum für Pädiatrische Rheumatologie am } \\
\text { Klinikum Stuttgart (ZEPRAS) }\end{array}$ \\
\hline 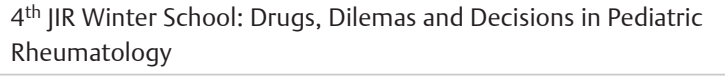 & 28.02.-01.03.2020 & Villars/Schweiz & $\begin{array}{l}\text { The Juvenile Inflammatory Rheumatism } \\
\text { Network }\end{array}$ \\
\hline 10. Trainingskurs in Kinder- und Jugendrheumatologie & 19. -21.03 .2020 & Leipzig & Initiative für das rheumakranke Kind e.V. \\
\hline 15. Kongress des Berufsverbandes Deutscher Rheumatologen & 27./28.03.2020 & Berlin & Berufsverband Deutscher Rheumatologen e.V. \\
\hline 23. Wörlitzer Expertengespräche & 24./25.04. 2020 & Wörlitz & Initiative für das rheumakranke Kind e. V. \\
\hline $\begin{array}{l}\text { 69. Jahrestagung der Norddeutschen Gesellschaft für Kinder- } \\
\text { und Jugendmedizin }\end{array}$ & 24./25.04.2020 & Celle & $\begin{array}{l}\text { Norddeutsche Gesellschaft für Kinder- und } \\
\text { Jugendmedizin }\end{array}$ \\
\hline $\begin{array}{l}\text { 37. Jahrestagung der Arbeitsgemeinschaft Pädiatrische } \\
\text { Immunologie }\end{array}$ & 07.-09.05.2020 & $\begin{array}{l}\text { Kloster Banz, } \\
\text { Burg Staffelstein }\end{array}$ & $\begin{array}{l}\text { Arbeitsgemeinschaft Pädiatrische Immuno- } \\
\text { logie (API) }\end{array}$ \\
\hline $\begin{array}{l}\text { EULAR } 2020 \text { - European League Against Rheumatism Annual } \\
\text { Congress }\end{array}$ & 03.-06.06. 2020 & Frankfurt & European League Against Rheumatism \\
\hline $\begin{array}{l}\text { Tag des rheumakranken Kindes und Jugendlicher (Fortbildung } \\
\text { für Ärzte) }\end{array}$ & 20.06 .2020 & Hamburg-Eilbek & $\begin{array}{l}\text { Hamburger Zentrum für Kinder- und Jugend- } \\
\text { rheumatologie }\end{array}$ \\
\hline $\begin{array}{l}\text { 48. DGRh-Kongress - Wissenschaftlicher Jahreskongress der } \\
\text { Deutschen Gesellschaft für Rheumatologie, 34. Jahrestagung } \\
\text { der Deutschen Gesellschaft für Orthopädische Rheumatologie } \\
\text { (DGORh) }\end{array}$ & 09.-12.09.2020 & München & $\begin{array}{l}\text { Deutsche Gesellschaft für Rheumatologie, } \\
\text { Deutsche Gesellschaft für orthopädische } \\
\text { Rheumatologie }\end{array}$ \\
\hline $\begin{array}{l}\text { 30. Jahrestagung der Gesellschaft für Kinder- und Jugendrheu- } \\
\text { matologie (GKJR), 116. Jahrestagung der Deutschen Gesellschaft } \\
\text { für Kinder- und Jugendmedizin (DGKJ) }\end{array}$ & 16. -19.09 .2020 & Berlin & $\begin{array}{l}\text { Deutsche Gesellschaft für Kinder- und } \\
\text { Jugendmedizin, Gesellschaft für Kinder- und } \\
\text { Jugendrheumatologie }\end{array}$ \\
\hline 26 $6^{\text {th }}$ European Paediatric Rheumatology Congress (PReS) & 23. -26.09 .2020 & $\begin{array}{l}\text { Prag/Tschechi- } \\
\text { sche Republik }\end{array}$ & The Pediatric Rheumatology European Society \\
\hline ACR/ARHP Annual Meeting & 06. -11.11 .2020 & $\begin{array}{l}\text { Washington } \\
\text { D.C./USA }\end{array}$ & $\begin{array}{l}\text { American College of Rheumatology/Associa- } \\
\text { tion of Reproductive Health Professionals }\end{array}$ \\
\hline $\begin{array}{l}13^{\text {th }} \text { Hamburg Symposium on juvenile Scleroderma - update on } \\
\text { new developments }\end{array}$ & 12./13.12.2020 & Hamburg-Eilbek & $\begin{array}{l}\text { Hamburger Zentrum für Kinder- und Jugend- } \\
\text { rheumatologie }\end{array}$ \\
\hline
\end{tabular}


tegiemeeting von Vorstand und Beirat der GKJR im März in Berlin stattfinden, auf dem die Ausrichtung der GKJR für die kommenden Monate diskutiert und festgelegt werden soll.

\section{Trainingskurs in Kinder- und Jugendrheumatologie}

Ebenfalls auf der diesjährigen Agenda steht der 10. Trainingskurs in Kinder- und Jugendrheumatologie vom 19. bis 21 . März 2020 in Leipzig. Initiiert wurde diese Veranstaltung für angehende Kinder- und Jugendärzte von der „Initiative für das rheumakranke Kind e. V.“. Themenschwerpunkte in diesem Jahr bilden Vorträge und Workshops zu rheumatologischen Differenzialdiagnosen nach Leitsymptom, zur evidenzbasierten Therapie und zur Autoinflammation. Darüber hinaus werden wichtige, bereits erarbeitete Themen praxisnah vertieft. In bewährter Weise stehen die praxisorientierten Se- minare in kleinen Gruppen im Mittelpunkt und werden durch Vorträge zu den Hauptthemen ergänzt. Als Referenten konnten führende deutsche Kinder- und Jugendrheumatologen gewonnen werden.

\section{ZUSATZINFO}

Die Teilnahmegebühr beträgt 250 Euro (Vorträge, Seminare, Kursmanuskript, 2x Hotelübernachtung mit Frühstück sowie Mittagessen am 2. Tag).

Formular zur Anmeldung: Webseite der GKJR (www.gkjr.de) unter Veranstaltungen.

Wegen der begrenzten Teilnehmerzahl werden die Anmeldungen in der Reihenfolge ihres Eingangs berücksichtigt.

\section{Gabriele Berg, Berlin}

\section{KONTAKTADRESSE}

Gesellschaft für Kinder- und Jugendrheumatologie

Geschäftsstelle

c/o Deutsches Rheuma-Forschungszentrum (DRFZ)

Programmbereich Epidemiologie

Gabriele Berg

Charitéplatz 1, 10117 Berlin

Tel.: 030/28 460-743

Fax: 030/28 460-744

E-Mail: gabriele.berg@drfz.de

IMPRESSUM

Verantwortlich für den Inhalt

Prof. Dr. Kirsten Minden

Universitätsmedizin Berlin -

Charité Campus Virchow und

Deutsches Rheuma-Forschungszentrum Berlin

Martina Niewerth, Deutsches

Rheuma- Forschungszentrum, Berlin 